\title{
ROL DE LOS TERAPEUTAS OCUPACIONALES EN EL TRABAJO CON PERSONAS EN SITUACIÓN DE CALLE EN CHILE: APORTES Y DIFICULTADES DESDE LA DISCIPLINA. UNA REVISIÓN BIBLIOGRÁFICA.
}

ROLE OF OCCUPATIONAL THERAPIST WORKING WITH HOMELESS IN CHILE: CONTRIBUTIONS AND CHALLENGES FROM THE DISCIPLINE. A LITERATURE REVIEW.

\section{Nataly Álvarez B. ${ }^{1}$ 1, Konny Álvarez S. 2, Catalina Berrocal B. 3, María Miranda C. 4, Daniela Olivares A.5}

\begin{abstract}
Resumen
La presente investigación tiene por objetivo establecer el rol de los Terapeutas Ocupacionales (T.O) dentro de políticas y programas de carácter público y privado, destinados a personas en situación de calle (PsC) en Chile. Este estudio cualitativo se basa en el enfoque de la Teoría Fundamentada, utilizando como estrategia de investigación, el análisis de artículos desde la disciplina que aborda el trabajo de la T.O con PSC en el extranjero, a través una revisión bibliográfica. Como resultados se obtuvieron siete categorías emergentes: comprensión del fenómeno, las causas que provocan esta realidad, el impacto de la intervención basada en la ocupación, los modelos teóricos utilizados desde la disciplina que respaldan la intervención, las capacidades, herramientas, funciones y dificultades de los Terapeutas Ocupacionales en el trabajo con esta población. Finalmente, se concluye que el rol del Terapeuta Ocupacional se orienta al desarrollo de habilidades necesarias para estos individuos considerando sus motivaciones e intereses, favoreciendo su inclusión social. Esto se relaciona con los valores y principios de igualdad resguardados en la Constitución de Chile (Ley 20.595) y con la visión de las entidades que trabajan con esta población.
\end{abstract}

Palabras claves:

Personas en situación de calle, rol del Terapeuta Ocupacional, Programas gubernamentales, Programas no gubernamentales.

\footnotetext{
1 Terapeuta Ocupacional. Universidad Austral de Chile. Nataly.alvarez.bizama@gmail.com

${ }^{2}$ Terapeuta Ocupacional. Universidad Austral de Chile. Konny.alvarez.saldivia@gmail.com

${ }^{3}$ Terapeuta Ocupacional. Universidad Austral de Chile. Catalina.berrocal.b@gmail.com

${ }^{4}$ Terapeuta Ocupacional. Universidad Austral de Chile. Leonor.miranda90@gmai.com

${ }^{5}$ Terapeuta Ocupacional, Universidad de Chile, Magister en Desarrollo a Escala Humana y Economía Ecológica @ , académica Universidad Austral de Chile. danielaolivares@uach.cl
} 


\begin{abstract}
The current research has the purpose of stablishing the role of Occupational Therapist (O.T) in policies and programs, both governmental and non-governmental, destined to Homeless in Chile. It was done through a qualitative view using a Grounded Theory methodology, through out a bibliographical review of articles from the discipline in regard with homeless and O.T issues. The findings arisen the following emerging categories: comprehension of the phenomenon, the causes of this situation, and the impact of interventions based on occupation, the theoretical models used from the discipline that supports the intervention, the skills, tools and purposes of O.T. and difficulties of O.T. working with this population. Finally, it is concluded that the role of the O.T. with this group of people is oriented towards the development of abilities needed to them effective tools for social reinsertion, based on the needs, motivation and interests considering their interests and motivations promoting social inclusion. These actions are relationship with the values and principles of equity guaranteed by the Political Constitution of Chile (Law N $\left.{ }^{\circ} 20.595\right)$ and the institutions working with this people.
\end{abstract}

\title{
Key Words:
}

Homeless, role of Occupational Therapist, Governmental Programs, Non-Governmental Programs.

\section{INTRODUCCIÓN}

La situación de calle es una realidad que ha estado presente por años en nuestro país, siendo abordada principalmente por Organizaciones no Gubernamentales (ONG's). Es a partir del año 2005 bajo el Gobierno de Ricardo Lagos (2000 - 2006) que este tema es reconocido como un problema por el Estado, realizándose el mismo año el Primer Catastro Nacional de Calle, cuyo objetivo fue contabilizar, dimensionar y caracterizar a las personas en situación de calle (de ahora en adelante PsC). Esto aportó información relevante para la elaboración e implementación de programas y políticas dirigidas a esta población, con el fin de facilitar su inclusión social y mejorar sus condiciones de vida. En esa oportunidad, se contabilizaron 7.254 PsC (Ministerio de Desarrollo Social, 2005).

Durante el Gobierno de Sebastián Piñera (20102014) se lleva a cabo el Segundo Catastro Nacional de Calle, el cual contabilizó 12.255 PsC. En él se identificaron diversas problemáticas de esta población, relacionadas con el trabajo, educación, salud, habitabilidad, causas, características y factores de permanencia en la situación de calle. Debido a esto, se materializan ejes de acción hacia este grupo de personas expresadas en el "Plan Nacional de Calle", explicado posteriormente (Ministerio de Desarrollo Social, 2012).

El año 2014 asume el Gobierno Michelle Bachelet, cuya visión permite complementar la percepción del Gobierno anterior en sus objetivos y ejes de acción. Actualmente, se encuentra en desarrollo el Programa Calle Chile Solidario, que apunta principalmente a que las personas cuenten con condiciones y recursos suficientes para mejorar su condición de vida en las áreas que ellos identifiquen como significativas (Ministerio de Desarrollo Social, 2014).

Para comprender el fenómeno es importante conocer la definición utilizada por las distintas entidades que trabajan en Chile con esta población. Las ONG's como Red Calle y el Hogar de Cristo, definen a las PsC como un fenómeno de carencia material que se relaciona con procesos de exclusión, desvinculación social y vulnerabilidad. Así, encontrarse en situación de calle involucra aspectos que van más allá de no contar con un techo o vivienda, ya que también se refiere a la carencia de un hogar como fuente de vinculación (Ministerio de Desarrollo Social, 2011).

Desde el Ministerio de Desarrollo Social (2011), órgano de Gobierno que se ha abocado al trabajo con este grupo, se entiende a las PsC como aquellos individuos sin alojamiento fijo y adecuado, que deben pagar o no a entidades públicas o privadas por un albergue temporal, ya sean residencias u hospederías solidarias o comerciales, "sin contar con una infraestructura que pueda ser caracterizada como vivienda, aunque ésta sea precaria" (p.11), no incluyendo a personas que vivan en campamentos.

Cabe destacar que desde el Gobierno, se diferencia a las personas en situación de pobreza y a las PsC o indigentes, mediante un método directo de necesidades básicas insatisfechas (NBI), el cual mide pobreza e indigencia en términos absolutos. Quiénes son pobres o indigentes y quiénes no lo son, se define en relación a mínimos de satisfacción de necesidades básicas, en el caso de la pobreza, o alimentarias, en el caso de la indigencia (CASEN, 2009)

El que existan personas que vivan bajo estas circunstancias, se debe a una serie de factores tanto per- 
sonales como contextuales que dan como resultado esta realidad. Marinkovic (2012) señala que algunas de las causas de la situación de calle son: la situación de orfandad y/o abandono, muerte de padres, ruptura del núcleo familiar (pareja, hijos), búsqueda de "libertad", necesidad de trabajar a temprana edad, alcoholismo, drogadicción, cesantía en largos e intermitentes periodos de tiempo, problemas de salud física y mental que les impiden trabajar, víctimas de abuso, de violencia y pobreza extrema. El Ministerio de Desarrollo Social (2014) incorpora dentro de las causas de la situación de calle, elementos contextuales del país que pueden propiciar el inicio de una vida en las calles.

Estas causas manifiestan las dificultades que presentan los participantes para mantenerse inmersos en su contexto (físico, social y cultural), lugar en el que ocupan una posición social. Respecto a esto Bourdieu plantea el concepto de campo social, como el "espacio social, formado por relaciones objetivas, entre posiciones diferenciadas, socialmente definidas y en gran medida independiente de la existencia física de los agentes que los ocupan" (Bourdieu, 1992, citado por Giménez, 1997 , p. 14). Por lo tanto, los "participantes" luchan con otros dentro de ese campo social global (dentro del cual están otros campos: económico, político, cultural, religioso, entre otros), por mantener u obtener su capital simbólico, es decir, prestigio, legitimidad y autoridad, a través de su modo de acción y pensamiento, lo que Bourdieu denomina habitus. Éste se relaciona con "aquello que hace que los agentes o personas dotados del mismo (habitus) se comporten de cierta manera en ciertas circunstancias" (Bourdieu, 1987, p. 40, citado por Giménez, 1997, p. 4), aspecto indisociable del concepto de campo y de capital.

En el momento que se enfrentan a la situación de caIle, comienzan a interactuar con otros "agentes" dentro de un campo social diferente. Por lo tanto, se enfrentan a situaciones nuevas, que para afrontarlas deben aprender la manera de actuar (habitus), para "sobrevivir" en este nuevo campo, el que según Max Neef (1986), es parte intrínseca del sistema económico capitalista.

El concepto de habitus ha sido abordado desde la Terapia Ocupacional (T.O) por Kielhofner (2011) en el Modelo de Ocupación Humana, quien incorpora la visión de Bourdieu, pero desde una perspectiva ocupacional, a través del concepto de habituación, entendido como un "patrón de comportamiento semi automático, que responde a nuestros contextos temporales, físicos y sociales conocidos" (p. 16).
Por esta razón, se espera que la intervención con este grupo de personas tenga un carácter complejo, relacionado con la motivación que éstos puedan tener para cambiar un estilo de vida al que ya están acostumbrados o habituados.

Frente a esta situación, no existen publicaciones en Chile sobre el trabajo realizado con este grupo de personas desde nuestra disciplina, lo que dificulta identificar y validar un rol claro del T.O en las políticas públicas y programas que abordan esta problemática.

En relación al concepto de "rol" éste será comprendido como "un conjunto de normas que definen la forma en que las personas de una posición social específica deben comportarse" (Myers, 2005, citado por González, González \& Vicencio, 2014, p. 143.); al especificarlo en un rol profesional, se relaciona con las funciones o conductas que desempeña el profesional y que esperan que desempeñe según su determinada profesión (Ander, 1995, citado por Alarcón \& Lai, 2007).

Desde nuestra disciplina, algunos autores dan cuenta de su experiencia con esta población y abordan la problemática vinculada a la motivación de este grupo. Kronenberg, Simó \& Pollard (2007) comprenden la situación de calle como un proceso gradual y agudo de desvinculación social, y no sólo una situación de pobreza material (falta de vivienda). Esta visión del problema surge de un programa de rehabilitación realizado en México con niños en situación de calle. En dicho estudio se percibió una falta de motivación por parte de los niños de salir de esta situación; según los autores "este reto estaba enraizado en las condiciones contextuales complejas y graves que constantemente privaron a esos niños/jóvenes de experiencias ocupacionales significativas y justas" (p. 265).

A partir de estos estudios, los autores desarrollan un nuevo concepto, apartheid ocupacional, el cual se define como la segregación de grupos de personas, al acceso de participación digna y significativa de ocupaciones de la vida diaria, poniendo en peligro su salud y bienestar (Kronenberg et al., 2007). Esta situación debe ser combatida mediante la inclusión social, entendida como:

El acceso de todos los ciudadanos a los recursos básicos, servicios sociales, oportunidades de empleo y derechos para participar plenamente en la vida económica, social y cultural y de disfrutar de un nivel de bienestar que se considera adecuado al tiempo y sociedad en la que se vive (European Comission, 2004, Citado por Fernández, Remy, Scott \& Carriazo, 2013, p. 7) 
Respecto al concepto de inclusión, éste se incorpora dentro del Plan Nacional de Calle, el cual surge desde la ley 20.595 (Ministerio de desarrollo Social, 2014). Dicho plan tiene por objetivo construir políticas públicas para esta población en particular, buscando la prevención de la situación de calle, protección de las personas en esta situación y la promoción social. Dentro de los programas ejecutados se encuentran el Programa de Apoyo a Adultos en Situación de Calle, Programa Noche Digna, Programa de Apoyo a Niños, Niñas y Adolescentes en Situación de Calle, Empleo con Apoyo, Programa de Atención en Salud Mental para PsC, Plan Invierno Calle y Centros para la Superación.

Para que las PsC puedan optar a los beneficios de los programas, es necesario que cumplan una serie de requisitos y compromisos de participación, para no ser excluidos de éste (Ministerio de Desarrollo Social, 2013). Las acciones de los programas señalados, brindan un servicio especializado de apoyo psicosocial, de forma que permitan conectar a estas personas con las redes.

Es así que entenderemos las intervenciones en red como:

Todas aquellas conversaciones/ acciones que implican un contacto directo de un equipo especializado de intervención con el sujeto (persona, pareja, familia, grupo). Tal como su nombre lo indica, la estrategia pasa por una intervención en la red del sujeto con el propósito de desarrollarla operativamente en función de los problemas y necesidades de éste, reconstruirla o sustituirla en caso de disfuncionamiento y de construirla cuando no existe (Martínez, 2004, p. 43)

Actualmente, en los programas del Gobierno se incluye la participación de profesionales del área de las Ciencias Sociales, Psicólogos y Terapeutas Ocupacionales. Nuestra labor se incorpora en un solo programa relacionado con el área de salud mental, el cual tiene la siguiente estructura: contacto inicial y aplicación de instrumentos donde realizan preguntas orientados a problemas de salud mental, de adicciones, entre otros. Si las personas desean participar, se firma una carta de compromiso y posteriormente comienzan las intervenciones con fechas predeterminadas (Ministerio de Desarrollo Social, 2013). A pesar que nuestra disciplina es incorporada en esta área, cabe cuestionarse si es posi- ble desempeñarnos dentro de otras áreas o prestaciones brindadas por el Estado.

Como se dijo en un inicio, las primeras instituciones a cargo del trabajo con PsC, fueron las ONG's y una de las más reconocidas en Chile, es el Hogar de Cristo (2011), la cual ha desarrollado su labor desde el año 1946, buscando disminuir la situación de vulnerabilidad y los daños asociados a las condiciones de calle, mediante servicios transitorios, reconociendo y potenciando las capacidades y recursos de estas personas. Las acciones de esta organización, se dirigen a fomentar la vinculación social (familia, grupos, comunidad, instituciones públicas), la inclusión y participación social, esperando que este grupo de personas, puedan generar proyectos personales que les permitan modificar su situación de calle (Hogar de Cristo, 2011).

El Hogar de Cristo (2011) cuenta con diferentes programas a lo largo de Chile, donde se destacan: las hospederías, programa de acogida, comedores fraternos, albergues, centros de encuentro de PsC y casas de acogida. Cada programa es implementado dependiendo de las necesidades de cada región del país.

Respecto a la visión del Hogar de Cristo, es posible evidenciar que los objetivos propuestos por esta organización son similares a los del Gobierno, no obstante, existe una contradicción entre las acciones realizadas y sus propios objetivos (Sziklai, s/f), debido a que sus servicios y prestaciones son principalmente de carácter asistencialista (Zulueta, 2008).

El asistencialismo se define como la falta de oportunidad para decidir y participar responsablemente en un contexto social, convirtiéndose en un objeto pasivo (Freire, 1970, citado por Zulueta, 2008). Esta situación provoca que las $\mathrm{PsC}$ que reciben los servicios de esta institución, se conviertan en meros receptores de prestaciones externas.

Es por esta razón que como T.O. concordamos con la visión de Paulo Freire en relación a que las personas se hagan agentes de su propia recuperación, posicionándose de forma crítica frente a sus problemas (Freire, $\mathrm{s}, \mathrm{f})$, dado que la participación activa de la persona en su tratamiento, determinará el valor y la efectividad de la terapia (Polonio, Durante \& Noya, 2001, p. 62)

Esta forma de intervención nace desde las bases disciplinares de la T.O, las cuales se fundamentan en un enfoque biopsicosocial y una visión holística hacia las personas. Esta perspectiva busca llegar a todos aquellos individuos que, por una u otra razón, vean dificultado su desempeño en actividades que tengan significado (Crepeau, Cohn \& Schell, 2005). 
Para cumplir con este objetivo, debemos enfocarnos en favorecer el bienestar y la salud de las personas, condiciones que se logran a través de la participación en ocupaciones significativas como el autocuidado, disfrutar de la vida (ocio) y contribuir al ámbito social y económico de la comunidad, es decir, productividad (Crepeau, et al., 2011). Entenderemos el concepto de bienestar como una evaluación subjetiva de la salud relacionada con sentimientos, tales como la autoestima y el sentido de pertenencia a través de la integración social. También es descrito como una sensación fisiológica agradable y deseable, que puede variar de una persona a otra (Wilcock, 1998).

Junto con esto, es importante considerar que la ocupación se da dentro de un ambiente específico o entorno, el cual se define según Kielhofner (2011) como las "características particulares físicas y sociales, culturales, económicas y políticas de los contextos personales que impactan en la motivación, la organización y el desempeño de la ocupación" (p. 86).

Para que los individuos puedan involucrarse sin restricciones en ocupaciones significativas, entendidas como un fenómeno social, sistémico, complejo y coherente con la justicia y el bienestar de las comunidades (Morrison, Olivares \& Vidal, 2011), es necesario que exista una condición de equilibrio entre justicia y bienestar al que tienen acceso los individuos dentro de su comunidad, en concordancia con sus necesidades.

De acuerdo con esto, la T.O incorpora el concepto de Justicia Ocupacional, que busca promover un cambio a nivel social y económico para incrementar la conciencia individual, comunitaria y política, a través del aumento de recursos y la igualdad de oportunidades, lo cual permite que los individuos alcancen su potencial y experimenten bienestar a través del desarrollo en ocupaciones (Kronenberg, et al., 2007)

Por esta razón es fundamental ampliar las acciones de los T.O en Chile hacia este grupo de personas, que como hemos visto, requiere de una intervención que apunte hacia una participación en ocupaciones significativas y de interés, favoreciendo las oportunidades en el elecciones ocupacionales, debido a que en la actualidad no existen prestaciones específicas con esta población que, como sabemos, presentan vulnerabilidad y dificultades para satisfacer sus necesidades básicas. Para esto, es necesario una reflexión al interior de la disciplina para comprender el fenómeno de PsC, y ofrecer una respuesta desde la T.O (Kronenberg, 2007), considerando que el "vivir en la calle no deja de ser una opción válida en una sociedad de derechos" (Sziklai, s,f, p. 4).

Por lo tanto, esta investigación tendrá por objetivo establecer el rol del T.O dentro de las políticas y programas gubernamentales y no gubernamentales dirigidas a PsC en nuestro país.

\section{Diseño Metodológico}

La presente investigación fue elaborada a través de un enfoque cualitativo, el cual se basa en métodos de recolección de datos sin medición numérica y sin conteo (Gómez, 2006); este tipo de investigación tiende a ser holística, ya que se esfuerza por comprender el fenómeno de interés y su entorno social de manera situada (Salamanca \& Martín-Crespo, 2007), lo que nos permitió identificar y relacionar información integral del trabajo con esta población desde nuestra disciplina.

El diseño de este estudio fue abordado desde la Teoría Fundamentada o Grounded Theory (Charmaz, 2006, citado por Puddephatt, 2006). Esta metodología enfatiza el descubrimiento y desarrollo de teorías, apoyado en un marco teórico y se utiliza con el objetivo de crear categorías a partir de datos y analizar las relaciones que se establecen entre ellas (Charmaz, 1990, citado por De la Cuesta, 2006). Este método, dinámico y flexible, nos permitió ampliar y/o generar una teoría acerca de la información con respecto al rol realizando un análisis constante del mismo, desde una perspectiva hermenéutica.

Para esta investigación, se realizó una búsqueda en textos tanto en inglés como en español propios de la disciplina, donde las palabras claves utilizadas fueron "homeless or bum or homelessness", "Occupational Therapy" or "Occupational Therapist" para los textos en inglés, y "personas en situación de calle", "Terapeuta Ocupacional" o "Terapia Ocupacional" para aquellos documentos en español. Estos términos claves debían estar presentes en el título del artículo, en el resumen y/o dentro de las palabras claves propias de los documentos.

La búsqueda señalada, fue llevada a cabo en las siguientes revistas de la disciplina: Occupational Therapy in Health Care, American Journal of Occupational Therapy, Australian Journal of Occupational Therapy, British Journal of Occupational Therapy, New Zealand Journal of Occupational Therapy, Occupational Therapy in Mental Health, siendo incluidos sólo aquellos 
documentos o artículos realizados entre los años 2000 a 2013. Esto permitió tener información actualizada referente al tema tratado. De la revista Chilena de Terapia Ocupacional, hasta la fecha Diciembre 2013, no se han publicado artículos relacionados con las "personas en situación de calle", lo que apoya nuestro supuesto de escasa información en Chile sobre el rol de la T.O con este grupo en particular.

Se determinó que los textos debían tratar sobre el trabajo de la T.O con PsC. En una primera instancia de selección, se trabajó con 18 textos, los cuales fueron traducidos en su totalidad y analizados. Posterior a la lectura de estos artículos, 11 fueron seleccionados, ya que cumplían con los objetivos de investigación, de los cuales se levantaron siete categorías emergentes: comprensión del fenómeno, causas de la situación de calle, impacto de la ocupación, modelos teóricos utilizados, capacidades y/o herramientas utilizadas por los Terapeutas Ocupacionales, dificultades de la Terapia Ocupacional y funciones de la Terapia Ocupacional.

\section{Resultados}

\section{Comprensión del Fenómeno}

Dentro de los artículos revisados se encontraron diferentes definiciones que permiten comprender qué se entiende por $\mathrm{PsC}$, existiendo diversas visiones y perspectivas de un mismo fenómeno.

La Coalición Nacional para Personas sin Hogar de EE.UU (NCH, 2002, citado por Finlayson, Baker, Rodman \& Herzberg, 2002; Griner, 2006) considera que las PsC son aquellos individuos que carecen de una residencia nocturna regular, adecuada y fija. Esta definición incluye a las personas que residen habitualmente en los albergues supervisados, vivienda temporal, o en un lugar que no está designado para dormir.

Junto con esto, parte de los artículos sostienen que la población sin hogar ha sufrido cambios a través de la historia, dado que por ejemplo, las personas que vivían en la calle en EE.UU eran principalmente hombres jóvenes o de mediana edad. Actualmente la proporción de mujeres, niños, y familias de la población sin hogar están en aumento constante (Finlayson, et al., 2002). Schultz-Krohn (2004), enfatiza el impacto que provoca en las familias el quedarse sin hogar, principalmente en las rutinas, organización y roles de cada individuo que conforma el sistema familiar, en especial roles parentales.

Otros artículos reconocen la complejidad de la "situación sin hogar" y las necesidades biopsicosociales de estas personas. Además mencionan que la alienación, privación de derechos en la comunidad, escasa motivación y la vida institucional contribuyen a la complejización de las necesidades de esta población, y que la exclusión social hace que las $\operatorname{PsC}$ se vean impedidos a acceder a servicios de atención (Bradley, Hersch, Reistetter \& Reed, 2011; Schultz-Krohn, 2004)

\section{Causas de la situación de calle}

La literatura demuestra la complejidad de determinar los factores que pueden predisponer a una persona a quedarse en situación de calle. De las razones que han surgido, se encuentran abuso de sustancias, síndrome de estrés postraumático y problemas de salud física. Estas causas no son excluyentes, por lo que las personas pueden experimentar un factor o varios al mismo tiempo (Bradley., et al., 2011).

Otros artículos centran las causas en factores de riesgo como la pobreza extrema, largos periodos de desempleo, la escasez de vivienda de interés social, desinstitucionalización y empleos en trabajos de baja categoría con salarios bajos (Applewaite, 1997; Citado por Finlayson, et al., 2002).

Asimismo, Grandisson, Mitchell-Carvalho, Tang, \& Korner-Bitensky (2009); Lloyd \& Bassett (2012), refieren que las personas que presentan problemas de salud mental tienen mayores probabilidades de vivir en la calle, y que cuyo estado de salud puede verse acentuado al vivir bajo éstas circunstancias. Se estima además que un $15-30 \%$, de la población sin hogar tiene una enfermedad mental grave y persistente, esquizofrenia o trastorno bipolar. Y que alrededor de un $20 \%$ tiene un doble diagnóstico de abuso de sustancias y enfermedades mentales graves (Finlayson, et al., 2002; Grandisson et al., 2009; Griner, 2006; Herzberg \& Finlayson, 2001; Parmenter, Barham \& Fieldhouse., 2013; Shordike \& Howell, 2002; Swenson, Bunch-Harrison, Brumbaugh, Kutty \& FitzGerald, 2005).

Por otra parte, algunos autores mencionan que el aumento en el número de mujeres y niños en situación de calle, se asocia a que muchos de ellos han sido víctimas de violencia doméstica (Herzberg \& Finlayson, 2001; Lloyd \& Bassett, 2012). 


\section{Impacto de la intervención basada en la Ocupación}

La participación de PsC en actividades significativas contribuye a la conexión que éstos puedan tener con la comunidad, lo que a su vez favorece la aceptación de un grupo social definido. Los beneficios de participar en actividades significativas han generado un impacto en cuatro temas específicos: el proceso de descubrimiento, sensación general de descubrir lo que son capaces de lograr, toma de decisiones, desviación de alcohol y otras adicciones, alivio de problemas de salud mental, el respeto y reconocimiento público, inclusión, aceptación e integración social (Thomas, Gray, McGinty \& Ebringer, 2011).

Estos autores refieren que los beneficios de participar en actividades significativas estimula una atracción inherente hacia el compromiso ocupacional, desarroIlando rutinas positivas que proporcionan organización, lo que es particularmente relevante para las personas sin hogar, debido a que, tienen pocos compromisos y rutinas para organizar sus vidas y ganar un sentido de bienestar (Thomas, et al., 2011).

En el mismo sentido, Schultz-Krohn (2004) agrega la importancia del involucramiento en rutinas significativas de las familias sin hogar, permitiendo amortiguar el impacto que genera la situación de calle al interior de ésta, favoreciendo la protección de sus miembros mediante un sentido de estabilidad. Esto se debe a que la realización de ocupaciones de interés, favorece el desarrollo de habilidades para la vida cotidiana, además de contribuir en la autopercepción, autoestima y el sentido de eficacia de los individuos (Swenson et al, 2005; Lloyd \& Bassett, 2012).

\section{Modelos teóricos utilizados desde T.O, que respaldan la intervención}

Las competencias de los T.O deben responder a las necesidades biopsicosociales de las $\mathrm{PsC}$, para ello se apoyan en diversos modelos teóricos para sustentar sus intervenciones; uno de éstos es el Modelo de Ocupación Humana (MOHO), el cual apoya el razonamiento profesional frente a los problemas arraigados, por ejemplo, alienación, vida institucional, escasa motivación y actitud defensiva contra el cambio (factores que contribuyen a la complejidad de necesidades de este grupo), evidenciándose en el reconocimiento que los hábitos y roles son naturalmente resistentes al cambio
(Lloyd \& Bassett, 2012; Parmenter, et al., 2013; Shordike \& Howell, 2002;), para ello los T.O estructuran sus intervenciones de acuerdo a tres niveles de motivación; Exploración, Competencia y Logro (Parmenter, et al., 2013).

Otro modelo teórico utilizado es el Modelo Canadiense de Desempeño Ocupacional (C.M.O.P). Dicho modelo es elegido por T.O debido a su énfasis en las actuaciones profesionales centradas en el cliente, la relación dinámica entre la persona, el medio ambiente, y su ocupación, y el hecho que identifica la espiritualidad como la esencia de la persona con componentes de desempeño (físico, cognitivo, afectivo) (Grandisson et al., 2009; Herzberg \& Finlayson, 2001), permitiendo conocer las necesidades de las PsC para el desempeño en distintas áreas de participación, generando intervenciones realistas y acordes a las vivencias e historia de vida de este grupo de personas, brindando además oportunidades de empoderamiento para tomar el control sobre sus vidas.

\section{Capacidades y/o Herramientas de los Terapeutas Ocupacionales}

Los T.O. tienen un enfoque único, que proporciona una visión y estrategias de intervención centrada en el cliente. Esto se debe a que tienen una formación académica distintiva que se enfoca en apoyar a las personas con discapacidad física y mental para que alcancen una mayor autonomía y participación en ocupaciones significativas, (Grandisson et al., 2009) minimizando los efectos residuales de abuso de sustancias, enfermedades mentales, la violencia doméstica, la baja autoestima y sentimientos de insuficiencia.

Otro autor menciona que algunas de las cualidades específicas de la T.O. para cumplir de manera efectiva con los objetivos de intervención son: el reconocimiento de la complejidad, eficacia de una fuerte conexión entre persona, ambiente y ocupación, además del desarrollo de la motivación. En relación a la conexión entre persona, ambiente y ocupación, los Terapeutas revelan la importancia del análisis de los entornos físicos y sociales de las PsC y en especial de los entornos institucionales (albergues), ya que estos ofrecen una experiencia de vida alternativa (Parmenter, et al., 2013), siendo fundamental la reconexión con la comunidad (Schultz-Krohn, 2004).

Para la pesquisa de necesidades de este grupo de personas las evaluaciones funcionales de T.O utiliza- 
das, incluyen una combinación de entrevistas, tareas prácticas y evaluaciones estandarizadas para ayudar en la identificación de los roles ocupacionales significativos (Lloyd \& Bassett, 2012; Swenson, et al., 2005). Además, Shordike \& Howell (2002) enfatizan la necesidad de realizar evaluaciones que permitan identificar las prioridades individuales de los usuarios, sus habilidades y fortalezas, para luego planificar un tratamiento individualizado.

\section{Dificultades de la Terapia Ocupacional}

A partir de la revisión de la literatura, se evidencia que los T.O tienen el conocimiento y las habilidades para trabajar con PsC (Heubner y Tryssenaar, 1996; 1999, citado por Herzberg \& Finlayson, 2001). No obstante, los miembros del personal y los residentes de hogares o albergues presentan un escaso conocimiento de la profesión (Finlayson, et al., 2002).

Gran parte de los investigadores que han trabajado extensamente con $\mathrm{PsC}$, tuvieron que atravesar diversas barreras administrativas. Un ejemplo de esta situación ocurre en una intervención desde T.O, donde se conecta a esta población con el uso del computador. Desde lo administrativo no se consideraba como prioridad realizar esta intervención, sin embargo, los T.O describen cómo el desarrollo de habilidades informáticas en torno a objetivos personales y de calificaciones de aprendizaje de la computadora fue crucial para crear sentimiento de competencia en estas personas (Swenson et al., 2005).

Asimismo, Grandisson et al., (2009); Shordike \& Howell (2002) señalan que, si bien los terapeutas logran reconocer y valorar su rol con este grupo de personas, a menudo no se sienten preparados para cumplir con el mismo, por ello plantean la necesidad de preparar a los estudiantes desde pre grado para las nuevas exigencias en esta área, específicamente en la comunitaria. Junto con ello, hacen hincapié en la necesidad de formación acerca de este tema tanto para médicos como otros profesionales del área que están ya en el campo.

De esta forma, con una mejor comprensión de los trabajos realizados por las $\mathrm{PsC}$, los profesionales de Terapia Ocupacional podrían ser capaces de aumentar el trabajo desde la disciplina con la población sin hogar. Además los terapeutas han reconocido la necesidad de participar activamente en la prestación de servicios para este grupo de personas, sin embargo, un mínimo de recursos están a disposición de los terapeutas para desarrollar intervención con esta población (Bradley et al., 2011).

\section{Funciones de la Terapia Ocupacional}

Uno de los objetivos claves desde nuestra disciplina es ayudar a los usuarios que se sienten excluidos, aislados, sin esperanza o sin valor a volver a ser parte de su mundo social, proporcionando oportunidades, utilizando las redes sociales y de apoyos importantes, tanto como sea posible (Grandisson et al., 2009; Lloyd \& Bassett, 2012).

El trabajo de nuestra disciplina se enfoca en las necesidades funcionales de las personas sin hogar, ayudándoles a asumir roles ocupacionales significativos e incorporar actividades de interés dentro de su rutina (Herzberg \& Finlayson, 2001; Schultz-Krohn, 2004). La inclusión de los T.O dentro de los albergues, ha permitido un enfoque holístico en la prestación de servicios, lo cual ha sido beneficioso para las personas que acceden a éstos (Grandisson, et al., 2009; Griner, 2006; Lloyd \& Bassett, 2012). El objetivo principal de la T.O es ayudar al usuario o cliente a maximizar las fortalezas y desarrollar habilidades para participar eficazmente en las actividades cotidianas, mediante la formación de habilidades para la vida, habilidades de trabajo, interpersonales, habilidades y manejo de dinero, entre otras (Lloyd \& Bassett, 2012).

Herzberg \& Finlayson (2001) plantean que mediante el análisis y adaptación de actividades, es posible desarrollar y adecuar intervenciones, que favorezcan el empoderamiento de las personas, permitiendo así remediar disfunciones ocupacionales que puedan resultar de una vida en las calles.

Para finalizar, Tryssenaar et al., (1999, citado por Herzberg \& Finlayson, 2001) sugiere que el desarrollo de programas desde Terapia Ocupacional en albergues para PsC tiene como objetivo la disminución de las barreras en la participación, indicando que el papel de la T.O está bien justificada para esta población, en la que se ha identificado el trabajo y la contribución a la sociedad como algo importante.

\section{DISCUSIÓN}

Como mencionamos anteriormente, en relación a las definiciones encontradas en torno al concepto de 
PsC, es posible evidenciar aspectos en los cuales la caracterización de este grupo de personas realizadas tanto por el Ministerio de Desarrollo Social, Hogar de Cristo, programas del Gobierno y artículos encontrados convergen, siendo el principal de éstos la falta de una vivienda física que sea estable o en otras palabras, una carencia de tipo material. No obstante, concordamos con la visión de la Red Calle, el Hogar de Cristo y algunos de los autores de los artículos encontrados como por ejemplo Schultz-Krohn (2004) quienes indican que esta realidad va mucho más allá que el no tener una estructura física en la cual cobijarse, por lo que para dar solución a esta situación no podemos limitar nuestros esfuerzos únicamente a satisfacer las necesidades de tipo material.

Debido a esto, consideramos que los aspectos incluidos en los artículos leídos y en los objetivos actuales del plan calle del Ministerio de Desarrollo Social, son absolutamente necesarios para una comprensión más amplia de este fenómeno, incluyendo conceptos tan importantes como los de exclusión, desvinculación social y vulnerabilidad, además de esclarecer la necesidad de entregar herramientas y desarrollar habilidades para lograr la reinserción social, visión que permite complejizar los procesos y estrategias a utilizar en el trabajo con este grupo de personas.

Para cumplir con este propósito, es necesario comprender que tal como se aprecia en la categoría II de los resultados, el vivir en la calle es una realidad multicausal. Por lo que esta situación no siempre comienza como una elección de vida personal, sino que es la consecuencia de múltiples factores en la que se incluye una estructura social o sistema definido, que no sólo impacta en la existencia de PsC sino que también en la falta de oportunidades de las mismas, causales de la exclusión social o apartheid ocupacional (Kronenberg et al, 2007) que llegan a experimentar.

Como ciudadanos, vivimos inmersos en un sistema de tipo capitalista por lo que, tal como plantea Bourdieu, nuestro orden social, estructura y/o sistema actual se da a partir de la interrelación de ciertos capitales sociales, culturales y económicos que nos permiten desenvolvernos en la sociedad. Para que este sistema económico y social capitalista se sustente a sí mismo, la existencia de grupos vulnerables se vuelve una necesidad (Max-Neff, 1986).

Esta realidad estructural a su vez determinaría la forma en la que se desenvuelven las PsC, además de los recursos, habilidades y oportunidades necesarias para sobrevivir dentro este contexto. La forma en que viven, interactúan y se desempeñan, se arraigan de tal forma que habitúan sus patrones de comportamientos según el campo social en el que están inmersos y según con los agentes que interactúan y así van a generar estrategias necesarias o modos de acción para desempeñarse efectivamente. Ya que estas personas están influenciadas por factores multidimensionales que les entrega la "vida en la calle", ya sea social, cultural, económico y simbólico, que muchas veces escapa a lo que llamamos sentido común.

Debido a esto, una de las dificultades que podríamos experimentar al momento de intervenir con este grupo de personas, es que al tratar de "normalizarlos" o incluirlos nuevamente en la sociedad, se corre el riesgo de volverlos "vulnerables", al sacarlos de un contexto en el que antes eran efectivos para insertarlos a uno que no conocen o simplemente no validan, por lo que de ser así, las probabilidades de que vuelvan a su entorno antiguo aumentan de forma considerable, ya que tal como refiere Kronenberg et al (2007), existe una falta de motivación por salir de esta situación, producto de las condiciones contextuales complejas en las que están inmersos.

Las habilidades y recursos personales varían y se diferencian de un usuario a otro, por lo que su intervención también cumplirá con esta característica. Desde la Terapia Ocupacional en el trabajo con PsC, se busca permitir el desempeño independiente en actividades que son significativas y placenteras, siendo relevante favorecer y potenciar su participación de acuerdo a sus intereses y la motivación que la persona presente, favoreciendo su bienestar en condiciones de justicia ocupacional.

Este enfoque único de la T.O busca una participación activa de los usuarios sobre sus propios cambios, sobre sus ocupaciones significativas y de interés y las metas que quieran realizar, co-construyendo el plan de intervención basado en negociación y centrado en el cliente, aspectos incorporados en el Modelo Canadiense de Desempeño Ocupacional. Este modelo es sensible a la realidad social de la actualidad, marcada por la marginación y exclusión de amplios sectores de la sociedad, discriminación por diversos motivos como económicos o por algún tipo de discapacidad.

El objetivo, entonces, es propiciar un cambio en las personas, de modo de facilitar la adaptación a sus circunstancias personales y contextuales, lo que se vuelve fundamental considerando la complejidad del trabajo con este grupo de individuos, puesto que no solo debemos enfrentarnos a las limitantes de la estructura social 
sino que además a características personales relacionados con los usuarios, específicamente a los cuadros de salud mental que éstos tienen asociados, entre los cuales se incluyen trastornos de personalidad, drogodependencia, depresión entre otros. Por lo que las características del equipo según la información analizada, apunta a un enfoque multidisciplinario, sin embargo consideramos pertinente y necesario lograr un enfoque interdisciplinario.

La visión actual del Gobierno y las políticas públicas dirigidas a las $\mathrm{PsC}$, se relacionan con la perspectiva de la disciplina, ya que se busca favorecer el desenvolvimiento autónomo y eficaz en los entornos propios de las $\mathrm{PsC}$, a través de las motivaciones personales, entregando las herramientas y estrategias necesarias para desempeñarse de manera efectiva en un nuevo medio, esto podría aumentar las posibilidades de mantenerse de forma definitiva en un nuevo contexto social.

Sin embargo consideramos que los protocolos de acción del plan calle son deficientes en aspectos motivacionales, puesto que procura que en un primer acercamiento las PsC se hagan conscientes de su situación y se comprometan a seguir un proceso de tratamiento basado en la completación de una entrevista y la firma de un compromiso (Ministerio de Desarrollo Social, 2013), lo cual se ve dificultado por las características personales de este grupo.

Por lo tanto, se busca desde la T.O lograr el bienestar y la salud a través de la participación en ocupaciones significativas, por ende, el objetivo final del terapeuta en el trabajo con este grupo de personas, debe estar enfocado en contribuir a la justicia ocupacional.

Dentro de nuestro país, las principales entidades que brindan un servicio a esta población, son de carácter no gubernamental, en el que si bien sus objetivos apuntan a una visión psicosocial sus acciones se basan principalmente en satisfacer las necesidades básicas de este grupo, reflejándose una actitud asistencialista por parte de estas instituciones, lo cual se contradice con las bases de la disciplina. A pesar de no estar incorporados dentro del equipo de trabajo del Hogar de Cristo, nuestros aportes serían altamente eficaces en concretar la visión de esta organización a partir de las herramientas y/o capacidades propias de la T.O, que incluye el trabajo en red.

Por otra parte, a través del análisis de los artículos y los programas del Gobierno y el Hogar de Cristo queda en manifiesto el escaso conocimiento y comprensión de la disciplina. Esta situación limita la participación y validación de nuestro trabajo como profesionales, tanto en programas gubernamentales como no gubernamentales.

Algunas de las limitaciones encontradas en nuestro estudio, fue la falta de información en cuanto al trabajo en esta área desde nuestra disciplina; además, el cambio de tendencia política del nuevo Gobierno producido durante el proceso de investigación (20132014), condujo a una actualización en la información de los programas destinados a este grupo de personas que no alcanzó a ser integrada en el estudio; asimismo, la imposibilidad de observar en terreno la implementación de programas, debido a que son llevados a cabo especialmente en la ciudad de Santiago, estando las investigadoras situadas en Valdivia. Es así que sugerimos para una futura investigación, realizar un estudio de orientación etnográfica de la implementación de estos programas a lo largo del país en base a las acciones del actual Gobierno recabando información relevante desde los y las T.O. que tengan experiencia en intervención con esta población. 


\section{RefERENCIAS BibLIOGRÁfiCAS}

Alarcón, P. \& Lai, R. (2007). Identidad, Roles Profesionales y Formación Académica en Trabajo Social: El caso de la Universidad De Magallanes. (Tesis de maestría. Universidad de Magallanes). www. umag.cl/biblioteca/tesis/alarcon_paz_2007.pdf [Consultado: Diciembre 2013]

Bradley, M., Hersch, G., Reistetter, T. \& Reed, K. (2011). Occupational Participation of Homeless People. Occupational Therapy in Mental Health. 27, (1), pp. 26-35. Doi: 10.1080/0164212X.2010.518311

Crepeau, E., Cohn, E., \& Schell, B., (2005). Willard y Spackman. Terapia Ocupacional. (10 ed.). Buenos Aires, Argentina: Médica Panamericana.

Crepeau, E., Cohn, E., \& Schell, B., (2011). Willard y Spackman. Terapia Ocupacional. ( $11^{\circ}$ ed.). Buenos Aires, Argentina: Médica Panamericana.

De la Cuesta, C. (2006). Teoría y Método: La teoría fundamentada como herramienta de análisis. http://193.145.233.67/dspace/bitstream/ 10045/876/1/culturacuidados_20_19.pdf [Consultado: Agosto 2013]

Finlayson, M., Baker, M., Rodman, L., \& Herzberg, G. (2002). The process and Outcomes of a Multimethod Needs Assessment at a Homeless Shelter. American Journal of Occupational Therapy, 56, pp. 313-321. Doi: 10.5014/ajot.56.3.313

Freire, P. (s/f). La educación como práctica de la libertad. http://laespiral. momoescuela.org/wp-content/uploads/2014/01/Educ-pract-libertad.pdf [Consultado: Agosto 2014]

Giménez, G. (1997). La sociología de Pierre Bourdieu. San Andrés Totoltepec.

Gómez, M. (2006). Introducción a la Metodología de la Investigación Científica. Córdoba: Editorial brujas.

González, M., González, I \& Vicencio., (2014). Descripción del rol autopercibido del psicólogo y sus implicancias en los procesos de formación de pregrado. Psicoperspectivas, 13, (1), pp. 108-120.

Grandisson, M., Mitchell-Carvalho, M., Tang, V., \& Korner-Bitensky, N. (2009). Occupational therapists' perceptions of their role with people who are homeless. The British Journal of Occupational Therapy, 72 (11), pp. 491-498. Doi: 10.4276/030802209X125776165 38672

Griner, K. (2006). Helping the Homeless: An Occupational Therapy Perspective. Occupational Therapy in Mental Health. 22, (1), pp. 4961. Doi: 10.1300/J004v22n01_04

Herzberg, G \& Finlayson, F. (2001). Development of Occupational Therapy in a Homeless Shelter. Occupational Therapy in Health Care. 13, (3/4), pp. 133-147. Doi: 10.1080/J003v13n03_11

Hogar de Cristo. (2011). Solidaridad en Acción. http://www.hogardecristo.cl/ [Consultado: Noviembre 2013]

Kielhofner, G. (2011). Modelo de Ocupación Humana: Teoría y Aplicación. $\left(4^{\circ}\right.$ ed). Buenos Aires: Panamericana.
Kronenberg, F., Simó,S., \& Pollard, N. (2007). Terapia Ocupacional sin fronteras. Aprendiendo del espíritu de supervivientes. Buenos Aires: Editorial Médica Panamericana

Lloyd, C. \& Bassett, H. (2012). The role of occupational therapy in working with the homeless population: An assertive outreach approach. New Zealand Journal of Occupational Therapy, 59 (1), pp. $18-23$

Marinkovic, F. (2012). Estudio en profundidad sobre las Personas en Situación de Calle pertenecientes a la ciudad de Talca. Tesis para optar al grado de Licenciada en Antropología, Universidad Austral de Chile.

Martínez, V. (2004). El trabajo en y con las redes. http://es.slideshare.net/ SOCIOLOGA/el-trabajo-en-y-con-las-redes [Consultado: Agosto 2014]

Max-Neef, M. (1986). La economía descalza: señales desde el mundo invisible. Buenos Aires:Nordan

Ministerio de Desarrollo Social. (2005) Habitando la Calle. Catastro Nacional de Personas en Situación de Calle. http://www.ministeriodesarrollosocial.gob.cl/ plancalle/docs/1.Primer_Catastro_Nacional_Personas_Situacion_Calle_2005.pdf. [Consultado: Noviembre, 2013]

Ministerio de Desarrollo Social (2010). Encuesta CASEN. http://www. ministeriodesarrollosocial.gob.cl/casen/definiciones/pobreza.html [Consultado: Noviembre 2013]

Ministerio de Desarrollo Social. (2011). Plan Calle. http://www.ministeriodesarrollosocial.gob.cl/plancalle/plan-calle/ [Consultado: Noviembre 2013]

Ministerio de Desarrollo Social. (2012). En Chile todos Contamos: segundo catastro nacional de personas en situación de calle. http:// www.ministeriodesarrollosocial.gob.cl/plancalle/docs/En_Chile_ Todos_Contamos.pdf [Consultado: Noviembre 2013]

Ministerio de Desarrollo Social. (2013). Subsistema Seguridades y Oportunidades: Normas Técnicas Plan Calle. Borrador de Documento de Gobierno.

Ministerio de Desarrollo Social. (2014). Informe de Desarrollo Social. http://www.ministeriodesarrollosocial.gob.cl/pdf/upload/ IDS_2014_2.pdf [Consultado: Noviembre 2013]

Ministerio de Desarrollo Social. (2014). Orientaciones para Gestión de Condiciones Mínimas: Programa Calle Chile Solidario. http:// www.ministeriodesarrollosocial.gob.cl/plancalle/documentos/ [Consultado: Agosto 2014]

Morrison, R., Olivares, D \& Vidal, D. (2011). La Filosofía de la Ocupación Humana y el Paradigma Social de la Ocupación. Algunas reflexiones y propuestas sobre epistemologías actuales en Terapia Ocupacional y Ciencias de la Ocupación. Revista Chilena de Terapia Ocupacional. 11, (2), pp. 102-119

Parmenter, V.,Basham, R \& Fieldhouse, J. (2013). An Occupational Therapy Intervention Service to hostels for homeless people: an overview. British Journal Occupational 76, (5), pp. 242-245. doi:10.427 6/030802213X13679275042807 
Polonio, B., Durante, P., Noya, B. (2001). Conceptos fundamentales de Terapia Ocupacional. Madrid: Médica Panamericana

Puddephatt, A. (2006). Special: An Interview with Kathy Charmaz: On Constructing Grounded Theory. Qualitative Sociology Review. 2 ,(3), pp. 6-20

Salamanca, A. \& Martín-Crespo, C. (2007). El diseño en la investigación cualitativa. http://www.nureinvestigacion.es/ficheros_ administrador/f_metodologica/fmetodologica_26.pdf [Consultado: Diciembre 2013]

Schultz-Krohn, W. (2004). The meaning of family routines in a homeless shelter. American Journal of Occupational Therapy, 58, pp. 531-542.

Shordike, A \&Howell,D. (2002).The Reindeer of Hope: An Occupational Therapy Program in a Homeless Shelter. Occupational Therapy in Health Care, 15, (1-2) pp.57-68. Doi:10.1080/J003v15n01_07.
Swenson K., Bunch-Harrison, S., Brumbaugh, B., Kutty, R., \& FitzGerald, K. (2005). The Meaning of Computers to a Group of Men who are Homeless. American Journal of Occupational Therapy, 59, pp. 191-197. doi:10.5014/ajot.59.2.191

Sziklai, A. s/f. Aproximación a la delimitación de los grupos de personas en situación de calle en la sociedad Chilena. Valdivia: Universidad Austral de Chile (no publicado).

Thomas, Y. Gray, M. McGinty S. \& Ebringer, S. (2011). Homeless adults engagement in art first steps towards identity, recovery and social inclusion. Australian Occupational Therapy Journal. 58, pp. 429436. Doi:10.1111/j.1440-1630.2011.00977

Wilcock, A. (1998). An occupational perspective of health. Thorofare: Slack.

Zulueta, S. (2008). Políticas Públicas y Privadas para Personas en Situación de Calle. Revista trabajo social (75), pp. 27-35 